\title{
Conservative treatment for patients with subacromial impingement: Changes in clinical core outcomes and their relation to specific rehabilitation parameters
}

\author{
Mikkel B Clausen Corresp., 1, 2,3 , Mikas B Merrild ${ }^{1}$, Adam Witten ${ }^{2}$, Karl B Christensen ${ }^{4}$, Mette K Zebis ${ }^{1}$, Per \\ Hölmich $^{2}$, Kristian Thorborg ${ }^{2,3}$ \\ 1 Department of Physiotherapy and Occupational therapy, Metropolitan University College Copenhagen, Copenhagen, Denmark \\ 2 Sports Orthopaedic Research Center - Copenhagen (SORC-C), Department of Orthopedic Surgery, Copenhagen University Hospital, Amager-Hvidovre, \\ Hvidovre, Denmark \\ 3 Physical Medicine and Rehabilitation Research-Copenhagen (PMR-C), Copenhagen University Hospital, Amager-Hvidovre, Copenhagen, Denmark \\ 4 Department of Biostatistics, University of Copenhagen, Copenhagen, Denmark \\ Corresponding Author: Mikkel B Clausen \\ Email address: mikkelbek@gmail.com
}

Background. Impaired patient-reported shoulder function and pain, external-rotation strength, abduction strength, and abduction range-of-motion (ROM) is reported in patients with subacromial impingement (SIS). However, it is unknown how much strength and ROM improves in real-life practice settings with current care. Furthermore, outcomes of treatment might depend on specific rehabilitation parameters, such as the time spent on exercises (exercise-time), number of physiotherapy sessions (physio-sessions) and number of corticosteroid injections, respectively. However, this has not previously been investigated.

The purpose of this study was to describe changes in shoulder strength, ROM, patient-reported function and pain, in real-life practice settings, and explore the association between changes in clinical core outcomes and specific rehabilitation parameters.

Methods. Patients diagnosed with SIS at initial assessment at an outpatient hospital clinic using predefined criteria's, who had not undergone surgery after 6 months, were included in this prospective cohort study. After initial assessment (baseline), all patients underwent treatment as usual, with no interference from the investigators. The outcomes Shoulder Pain and Disability Index (SPADI:0-100), average pain (NRS:0-10), external rotation strength, abduction strength and abduction ROM, pain during each test (NRS:0-10), were collected at baseline and at six month follow-up. Amount of exercise-time, physio-sessions and steroid-injections was recorded at follow-up. Changes in outcomes were analyzed using Wilcoxon Signed-Rank test, and the corresponding effect sizes (ES) were estimated. The associations between changes in outcomes and rehabilitation parameters were explored using multiple regression analyses.

Results. Sixty-three patients completed both baseline and follow-up testing. Significant improvements were seen in SPADI (19 points, ES:0.53, $p<0.001$ ) and all pain variables (median 1-1.5 points, ES:0.26$0.39, p<0.01)$, but not in strength and ROM (ES:0.9-0.12, p>0.2). A higher number of physio-sessions was significantly associated with larger improvements in external rotation strength $(0.7$ Newton/session, $\mathrm{p}=0.046)$, and higher exercise-time was significantly associated with decrease in average pain $(-0.2$ points/1000min., $p=0.048$ ).

Discussion. Patient-reported function and pain improved after six months of current care, but strength 
and ROM did not improve. This is intersting, as strengthening exercises is part of most current interventions. While two significant associations were identified between self-reported rehabilitation parameters and outcomes, the small gains per physio-session or $1000 \mathrm{~min}$ of exercise-time reduces the clinical relevance of these relationships. Collectively, the findings from this study indicate room for improvement of the current rehabilitation of SIS, especially with regard to core clinical outcomes, such as strength and range of motion. 
1 Title

2 Conservative treatment for patients with subacromial impingement: Changes in clinical

3 core outcomes and their relation to specific rehabilitation parameters

4

5 Clausen $\mathrm{MB}^{1,2,3}$, Merrild MB${ }^{1}$, Witten $\mathrm{A}^{2}$, Christensen $\mathrm{KB}^{4}$, Zebis $\mathrm{MK}^{1}$, Hölmich $\mathrm{P}^{2}$, Thorborg $6 \mathrm{~K}^{2,3}$

7 1) Department of Physiotherapy and Occupational Therapy, Faculty of Health and Technology, 8 Metropolitan University College, Copenhagen N, Denmark.

9 2) Sports Orthopaedic Research Center - Copenhagen, Department of Orthopedic Surgery,

10 Copenhagen University Hospital, Amager-Hvidovre, Denmark.

11 3) Physical Medicine and Rehabilitation Research - Copenhagen (PMR-C), Amager-Hvidovre

12 Hospital, Copenhagen University Hospital, Copenhagen, Denmark

13 4) Department of Biostatistics, University of Copenhagen, Copenhagen, Denmark

14

15

16

17

18

19

20

21

22

23

\section{Corresponding author}

Mikkel Bek Clausen

Department of Physiotherapy and Occupational therapy, Metropolitan University College

Sigurdsgade 26, DK-2200 Copenhagen N, Denmark

Email: mikkelbek@gmail.com 
24 Background. Impaired patient-reported shoulder function and pain, external-rotation strength,

25 abduction strength, and abduction range-of-motion (ROM) is reported in patients with

26 subacromial impingement (SIS). However, it is unknown how much strength and ROM improves

27 in real-life practice settings with current care. Furthermore, outcomes of treatment might depend

28 on specific rehabilitation parameters, such as the time spent on exercises (exercise-time), number

29 of physiotherapy sessions (physio-sessions) and number of corticosteroid injections,

30 respectively. However, this has not previously been investigated.

31 The purpose of this study was to describe changes in shoulder strength, ROM, patient-reported

32 function and pain, in real-life practice settings, and explore the association between changes in

33 clinical core outcomes and specific rehabilitation parameters.

34 Methods. Patients diagnosed with SIS at initial assessment at an outpatient hospital clinic using

35 predefined criteria's, who had not undergone surgery after 6 months, were included in this

36 prospective cohort study. After initial assessment (baseline), all patients underwent treatment as

37 usual, with no interference from the investigators. The outcomes Shoulder Pain and Disability

38 Index (SPADI:0-100), average pain (NRS:0-10), external rotation strength, abduction strength

39 and abduction ROM, pain during each test (NRS:0-10), were collected at baseline and at six

40 month follow-up. Amount of exercise-time, physio-sessions and steroid-injections was recorded

41 at follow-up. Changes in outcomes were analyzed using Wilcoxon Signed-Rank test, and the

42 corresponding effect sizes (ES) were estimated. The associations between changes in outcomes

43 and rehabilitation parameters were explored using multiple regression analyses.

44 Results. Sixty-three patients completed both baseline and follow-up testing. Significant

45 improvements were seen in SPADI (19 points, ES:0.53, $\mathrm{p}<0.001)$ and all pain variables (median

46 1-1.5 points, ES:0.26-0.39, p<0.01), but not in strength and ROM (ES:0.9-0.12, p>0.2). A higher

47 number of physio-sessions was significantly associated with larger improvements in external

48 rotation strength ( 0.7 Newton/session, $\mathrm{p}=0.046)$, and higher exercise-time was significantly

49 associated with decrease in average pain (-0.2 points $/ 1000 \mathrm{~min} ., \mathrm{p}=0.048)$.

50 Discussion. Patient-reported function and pain improved after six months of current care, but

51 strength and ROM did not improve. This is interesting, as strengthening exercises is part of most

52 current interventions. While two significant associations were identified between self-reported

53 rehabilitation parameters and outcomes, the small gains per physio-session or 1000 min of

54 exercise-time reduces the clinical relevance of these relationships. Collectively, the findings

55 from this study indicate room for improvement of the current rehabilitation of SIS, especially

56 with regard to core clinical outcomes, such as strength and range of motion. 


\section{Introduction}

59 Subacromial impingement syndrome (SIS) is the most common shoulder disorder [1]. The

60

61

62

63

64

65

66

67

condition is characterized by shoulder pain and impaired patient-reported function [2], but

reduced strength and range of motion (ROM) has also been reported in patients with SIS $[3,4]$.

More specifically, abduction and external rotation strength, as well as abduction ROM, is only

two-thirds of that in the unaffected shoulder [3] or healthy controls [4], which is why a $\sim 50 \%$

increase is needed to reach normal strength and ROM levels. Based on this, it is suggested that patient-reported function, pain, strength and ROM should be considered core outcomes related to SIS [3].

Conservative treatment, including exercise therapy, is considered the first line of treatment $[5,6]$, and a range of randomized controlled trials (RCTs) show promising improvements in patientreported shoulder function, pain and ROM as a result of exercise therapy [7-18]. However, when maximum shoulder strength is measured, improvements vary, ranging from $4-36 \%$ in external rotation strength (derived from $[8,9,16,18-21]$ ) and 4-42\% in abduction strength (derived from $[8,9,16,18-22])$. Importantly, however, strength improvements seem to be smallest in studies including patients whose duration of symptoms is greater than two months. In these studies, strength improvement ranges from $4 \%$ to $15 \%$ in external rotation strength and $4 \%$ to $20 \%$ in abduction strength (derived from $[9,18,19,21]$ ). This is far from the $\sim 50 \%$ increase needed to restore the above-mentioned shoulder strength deficits reported by MacDermid et al. [4] and Clausen et al. [3]. It should also be noted that both the previously reported improvements in patient-reported shoulder function, pain and ROM, as well as the less encouraging changes in strength, were all achieved in clinical trial settings. While such studies have high internal 
81 validity, it is possible that the observed improvements could partly be due to a trial effect, such

82 as the Hawthorne effect and/or placebo effect [23], and hence it is possible that results obtained

83 outside such controlled settings will be less encouraging.

85 Conservative treatment strategies often include home-based exercises $[17,18]$. The effectiveness

86 of such exercise interventions likely depends on patients' adherence to prescribed exercises,

87 although this has not been demonstrated in patients with SIS. This is important because

88 utilization of exercise therapy in the rehabilitation of patients with shoulder disorders might be

89 limited, as indicated in a recent register-based study of 57,311 Danish shoulder patients in

90 secondary care (70\% with SIS), of whom only $43 \%$ had physiotherapy within 52 weeks after the

91 first hospital visit, and only $62 \%$ of physiotherapy sessions included exercise therapy or advice

92 on self-training [24]. This indicates a low utilization of some specific physiotherapy and

93 rehabilitation parameters, such as exercise therapy, potentially leading to suboptimal

94 rehabilitation for many patients. In addition to that, the actual time that patients with SIS spend

95 on exercise therapy has not been investigated and is therefore currently unknown. In relation to

96 physiotherapy and exercise therapy, it is also unknown to what degree the number of completed

97 physiotherapy sessions and the time spent on exercise therapy are related to improvements in the

98 SIS-related core outcomes, such as shoulder strength, ROM, patient-reported function and pain

$99[6,25]$. 
101 Knowledge about improvements in SIS-related core outcomes following rehabilitation and their

102 relationship to specific rehabilitation parameters will help researchers and clinicians understand 103 to what degree these clinical core outcomes are addressed in current care.

\section{Purpose:}

The purposes of this study were: first, to describe the changes in shoulder core outcomes, including strength, ROM, patient-reported shoulder function and pain, from baseline to six months follow-up, in patients diagnosed with SIS at the baseline examination at a public hospital outpatient clinic; second, to describe specific rehabilitation parameters, such as the utilization of physiotherapy and the amount of home-based rehabilitation performed during the same period; third, to investigate the association between shoulder core outcomes and specific rehabilitation parameters.

\section{Materials and Methods}

115 This is a prospective cohort study based on the six-month follow-up of 129 SIS-patients (82\%)

116 from a consecutive cohort of 157 SIS-patients [3]. At baseline, patients underwent a clinical

117 assessment of shoulder strength, ROM, patient-reported shoulder function and pain impairments

118 performed by one of six trained assessors (two physiotherapists, three physiotherapy

119 undergraduates and one medical student). After these assessments, a clinical examination was

120 performed by an orthopaedic surgeon shoulder specialist blinded to the results of the baseline

121 assessments. After baseline, all patients underwent usual care in settings not provided or

122 controlled by the investigators of this study. Accordingly, the content and progression of care 
123 was based solely on the choices of the doctors, therapists and patients, with no interference from

124 any investigators or other intruding parties.

126 Approximately six months after baseline (median 28 weeks [IQR: 27; 29]), patients included in

127 the consecutive cohort of SIS-patients were contacted by telephone, and a ten-minute interview

128 regarding treatment and rehabilitation since baseline was conducted. Information about sick

129 leave due to the shoulder disorder was also obtained but not presented in this study. Patients who

130 had not undergone shoulder surgery were invited to a follow-up assessment of maximum

131 isometric shoulder strength, ROM, patient-reported function and pain, using the same procedures

132 as in the baseline examination. Follow-up assessments were performed by one of five assessors

133 (physiotherapy undergraduates) who did not participate in the baseline assessment, but were

134 trained by the primary investigator $(\mathrm{MBC})$ and one of the assessors involved in the baseline

135 examinations. Follow-up assessments were conducted at the same facility as the baseline

136 assessments. Only the affected shoulder was tested at the follow-up examination. The study has

137 been evaluated by the Capitol Region Committee on Health Research Ethics in Denmark, where

138 it was evaluated as not requiring formal ethical approval (H-3-2013-FSP29). Written informed

139 consent was obtained from all participants.

\section{Participants}

142 Inclusion and exclusion criteria for the consecutive cohort of 157 patients with SIS, who form

143 the basis of this follow-up study, have been described in more detail in a previous paper [3].

144 Patients were considered eligible for inclusion based on the following criteria: age 18 years or 
145 more, sufficient Danish language ability, referred to examination of a shoulder problem and at

146 least three of the five diagnostic tests for SIS were positive (Hawkins-Kennedy, Neer's, painful

147 arc, Resisted External Rotation and Jobe's), as described by Michener et al. [26]. Patients were

148 excluded based on the following criteria: Presence of 1) a full thickness rotator cuff tear, luxation

149 or sub-luxation of the glenohumeral or the acromioclavicular joint, frozen shoulder or

150 osteoarthritis in the glenohumeral joint (based on clinical and/or paraclinical examination), 2) a

151 labral lesion verified by paraclinical investigation, or 3) any competing disorder affecting the

152 shoulder function or the ability to answer patient-reported questionnaires (e.g. neurological

153 disease, cervical disorder, elbow disorder, mental disorder or blindness).

154

155

156

157 Core outcome measures

158 SPADI

159 The Shoulder Pain and Disability Index (SPADI) was used to measure patient-reported shoulder

160 function. SPADI consists of 13 items, each scored on an 11-point numeric rating scale. The two

161 domains pain (five questions) and disability (eight questions) are scored separately from 0

162 (worst) to 100 (best), and averaged into a total SPADI score. The Danish version of SPADI is

163 considered reliable and valid [27]. 
166 Active abduction ROM was measured in degrees using a digital inclinometer. First, with the

167 subject standing with the arm in the anatomical position and the elbow extended, the

168 inclinometer was reset on a vertical surface. The subject raised the arm in the coronal plane

169 towards the ceiling and a measurement was taken with the inclinometer aligned parallel to the

170 humerus, close to the insertion of the deltoid muscle. The inter-tester reliability of the test is

$171 \operatorname{good}($ ICC 0.95) [28].

173 Strength

174 Maximum isometric peak force in abduction and external rotation was assessed with the shoulder

175 in neutral position and the elbow fully extended or flexed to 90 degrees. The subject was seated

176 close to a wall, which was used as external resistance to the isometric contraction. Measurements

177 were obtained using a hand-held dynamometer and measured in newtons $(\mathrm{N})$. This method has

178 been shown to have a high inter-tester reliability $\left(\mathrm{ICC}_{2,1}>0.9\right)$ [3]. Detailed descriptions of

179 assessment procedures are freely available in the online supplement to a previous study [3].

181 Patient characteristics and specific rehabilitation parameters

Demographics and disease-specific characteristics, including severity

183

184

185

186
The following disease specific characteristics were collected at baseline: Duration (duration of current shoulder problem: 0-1 months, 1-3 months, 3-6 months, >6 months); Sick Leave (on sick leave or unemployed due to shoulder problem, yes/no); Affected Side (dominant side/nondominant side diagnosed with SIS); Age (years); and gender (male/female). 
Information on shoulder surgery, corticosteroid injections, physiotherapy and time spent on exercises was based on the questions from the structured telephone interview (Table 1). First,

patients who answered 'yes' to having had shoulder surgery (Q1) were categorised as having had surgery since baseline, and were not asked further questions regarding their treatment since baseline. The number of corticosteroid injections and physiotherapy sessions received since baseline was determined through the answer to question Q2 and Q3, respectively. Finally, total minutes of exercises was calculated as the number of weeks with exercises multiplied by the average time spent on exercises per week with exercises $(\mathrm{Q} 5 * \mathrm{Q} 6)$.

Information on patient-reported recovery was collected from answers to the question "Is your shoulder disorder fully cured?" (yes/no). Improvement since baseline (Global impression of change, GIC) was collected through answers to the following question using a seven-point Likert scale: "How do you perceive your shoulder disorder now as compared to when you underwent the first shoulder examination six months ago?" Much worse, a very important aggravation; Worse, an important aggravation; Slightly worse, but enough to be an important aggravation; The same; Slightly better, but enough to be an important improvement; Better, an important improvement; Much better, a very important improvement). The severity of the shoulder disorder 
208 information on GIC and severity was collected through a self-developed, standardized, self-

209 administered questionnaire.

210

\section{Data reduction and statistics}

212 Demographics, disease specific information, baseline values for all outcomes and descriptive

213 data on the treatment received since baseline are presented as means $\pm \mathrm{SD}$, median $[\mathrm{IQR}]$ or

214 numbers and proportions, for conservatively-treated patients who underwent follow-up

215 assessment and those who did not, respectively. Age was compared between groups using

216 independent samples t-test; proportions were compared using $\mathrm{Chi}^{2}$-test or Fisher's exact test as

217 appropriate; baseline values, number of physiotherapy sessions and total minutes of home

218 exercises were compared using the Mann-Whitney U test.

220 For patients who underwent follow-up assessment, SPADI-score, strength, ROM, pain values

221 and self-rated severity at baseline and follow-up, as well as changes from baseline to follow up,

222 are presented as both mean \pm SD and median [IQR]. Change scores were calculated as the value

223 at follow-up minus the value at baseline. A Wilcoxon signed-rank test was applied in order to

224 determine whether changes were significant, and corresponding effect sizes (ES) were calculated

225 as the test statistic divided by the number of observations $\left(\mathrm{ES}=\frac{Z}{\sqrt{n}}\right)$.

227 Separately for each outcome (SPADI-score, external rotation strength, abduction strength,

228 abduction ROM, pain during each test and average pain level), the association between the

229 change in the outcome (dependent variable) and the number of physiotherapy sessions, the time 
230 spent on home exercises and the number of steroid injections (independent variables) were

231 investigated using multiple regression analysis. These exploratory multivariate regression

232 analyses were performed separately for each pair of dependent and independent variables,

233 including the baseline value of the dependent variable in question as covariate.

234 A significance level of 0.05 was applied for all statistical tests.

\section{Results}

237 From the original consecutive cohort of 157 patients with SIS, $129(82 \%)$ completed the

238 standardized telephone interview. Thirty-one of these had undergone surgery for their shoulder 239 disorder since the baseline examination, leaving 98 conservatively-treated patients who were all

240 invited to follow-up assessment. Sixty-three of the 98 conservatively-treated patients participated

241 in follow-up assessment approximately six months after baseline. Demographics, disease specific

242 information and baseline values for all outcomes are presented in Table 2, separating the

243 conservatively-treated patients that participated in follow-up assessment $(n=63)$ from those who

$244 \operatorname{did} \operatorname{not}(\mathrm{n}=35)$. For further details on study flow, see Figure 1.

246 The group of patients participating in the follow-up assessment had significantly improved from

247 baseline to follow-up in SPADI-score (median 19 points, [3; 39], ES 0.53, $\mathrm{p}<0.001$ ) and all pain

248 variables (median 1 to 1.5 points on NRS, ES 0.26 to $0.39, \mathrm{p}<0.01$ ), with changes in average

249 pain being the most pronounced. No changes were found in external rotation strength (median 2

$250 \mathrm{~N}[-9 ; 17]$, ES 0.09, p=0.337), abduction strength (median $4 \mathrm{~N}[-10 ; 16], \mathrm{ES} 0.12, \mathrm{p}=0.223$ ) and

251 Abduction ROM $\left(-1^{\circ}[-12 ; 30]\right.$, ES 0.09, p=0.324). See Table 3 for further details. 
253 At follow-up, 25\% (16 of 63) rated themselves as fully recovered or much improved. The current 254 severity of the shoulder disorder was rated $\geq 3$ on a $1-5$ scale ( 0 is best, 5 is worst) by $46 \%$ ( 29 of

255 63) at follow-up. For further details on global impression of change and severity at follow-up,

256 see Figures 2 and 3. From the conservatively-treated patients that participated in follow-up

257 assessment $79 \%$ reported having had $0-1$ steroid injections, $75 \%$ had received physiotherapy

258 (median 5 sessions [IQR 0; 11] for the entire group) and 87\% had performed exercises for their

259 shoulder disorder (median total exercise time of 1,040 minutes [IQR 220; 2,700] for the entire

260 group). The conservatively-treated patients who did not participate in follow-up assessment

261 reported significantly lower total exercise time compared to the conservatively-treated patients

262 who did participate in follow-up assessment $(\mathrm{p}=0.045)$, while the other rehabilitation parameters

263 listed in Table 4 did not differ significantly between groups. See Table 4 for further details on

264 treatment since baseline.

In the multivariate regression analyses of the results from the follow-up assessment of patients, significant positive associations were identified between the number of physiotherapy sessions and external rotation strength improvements, $+0.7 \mathrm{~N} /$ session $(95 \% \mathrm{CI} 0.0$ to $1.3, \mathrm{p}=0.046)$ as well as exercise-time and improvements in average pain, -0.2 points on an 11-point NRS scale per $1000 \min (95 \% \mathrm{CI}-0.4$ to $0.0, \mathrm{p}=0.048)$. No additional significant associations between changes

271 in outcome from baseline to follow-up and the total minutes of home exercises, the number of

272 physiotherapy sessions, or the number of corticosteroid injections, were identified ( $\mathrm{p}>0.05)$, see

273 Tables 5 and 6 for further details. 


\section{Discussion}

276 In this prospective study of patients treated conservatively for SIS, medium to large effect sizes

277 were seen for improvements in subjective outcomes of function and pain, approximately six

278 months following initial assessment, in 63 conservatively treated SIS-patients. Interestingly,

279 objective measures of strength and ROM did not improve.

\section{Changes in strength and ROM}

282

283

To the best of our knowledge, it has not previously been reported to what extent shoulder strength and ROM have changed in patients with SIS who underwent conservative treatment outside a controlled clinical trial setting. Therefore, the most important finding of this study is that usual care did not improve these core clinical outcomes assessing important impairments such as strength and mobility, with non-significant median changes in glenohumeral strength and shoulder abduction ROM close to zero ( -1 degree and 2 to $4 \mathrm{~N}, \mathrm{p}>0.2)$. This is despite the fact that shoulder mobility and strengthening exercises aimed at the rotator cuff are a part of most treatment programs $[12,17,18,22]$. Such lack of improvement is especially relevant considering that the same group of patients have been shown to lack $\sim 50 \%$ in abduction ROM as well as abduction and external rotation strength, to reach the same level as the unaffected shoulder [3].

Before our study, data regarding improvements in glenohumeral strength in patients with SIS, who had received an intervention including rotator cuff strengthening exercises, have solely been available from clinical trials, revealing somewhat varying results. Accordingly, external rotation strength $[8,16,19-21]$ and abduction strength $[8,16,19,21]$ improved significantly in some of the 
297 trials, with improvements ranging from 15\% (derived from [21]) to 43\% (derived from [8]). In

298 five clinical trials, strength in abduction $[9,20,22]$ and external rotation $[9,18]$ did not change

299 significantly. Only in the two trials by Dilek et al. [8] and Galaca de Freitas et al. [20] did

300 average improvements in abduction and/or external rotation strength exceed $20 \%$. The trial by

301 Dilek et al. [8] in particular stands out, not only with pronounced strength improvements in both

302 abduction (43\%) and external rotation (32\%), but also near-perfect outcomes in pain scores, with

303 minimal-to-no pain at 12-weeks follow-up for patients who had received an intervention

304 including rotator cuff strengthening exercises. While these treatment outcomes are impressive, 305 they indicate that the population included by Dilek et al. [8] might not be comparable to that of

306 other studies, including the current study, so the encouraging results regarding increases in

307 shoulder strength may not represent the typical response pattern. In support of Dilek et al. large

308 improvements $(36 \%)$ in external rotation strength were also found in the trial by Galaca de

309 Freitas et al. [20]. In that trial, the population seems to mirror the population included in the

310 present study and most of the aforementioned trials that included shoulder strength as an

311 outcome $[9,16,18,19,21,22]$, indicating that pronounced strength improvement may be possible

312 in these populations, but is often not achieved. In summary, based on the findings from our study

313 and previous studies, it appears that current treatment strategies in the available scientific

314 literature often do not address shoulder strength impairments sufficiently in patients with SIS,

315 and future research should aim to improve the rehabilitation of these impairments.

317 The non-existent change in abduction ROM between baseline and follow-up $\left(-1^{\circ}\right.$ [IQR $\left.-12 ; 30\right]$,

$318 \mathrm{p}=0.324)$ in this study, with the median at follow-up reaching only $137^{\circ}[\mathrm{IQR} 107 ; 153]$, is in

319 contrast to the more encouraging results found in patients with SIS who underwent active 
320 treatment with shoulder exercises in clinical trial settings. In these trials, improvements of $>20$

321 degrees [7,9] or follow-up results close to 180 degrees $[8,10]$ were reported for groups with

322 varying starting levels of abduction ROM. Interestingly, the lack of improvement in abduction

323 ROM found in our study is comparable to the treatment results in control group patients on the

324 waiting list for two months in the RCT study by Lombardi et al. [9]. Accordingly, it seems that

325 the results of current care, regarding the core outcome of shoulder abduction ROM, are a long

326 way from matching the encouraging results found in clinical trials. While this does question the

327 effectiveness of current care in the rehabilitation of patients with SIS, it also indicates an

328 important difference in treatment responses obtained in clinical trials compared to real life

329 settings. This apparent difference in treatment response underlines the importance of conducting

330 RCTs using a more pragmatic approach to obtain valid data on the target group regarding the

331 effectiveness of rehabilitation interventions. Such an approach would help inform both clinicians

332 and policy makers when recommending or implementing specific rehabilitation strategies.

Changes in patient-reported function, pain, global impression of change and severity

335

336

337

338

339

340

341

342

In contrast to the objective outcomes of shoulder strength and ROM, both patient-reported function and all pain outcomes improved significantly in the current study (mean SPADI improvement of 23 points, median pain improvements of 1-1.5 points on 11-point NRS). This improvement in SPADI score is comparable to the mean improvement of 22.4 points after 22 weeks reported by Bennell et al. [18], the 23.5 and 29.1 points (control and intervention group, respectively) after six months reported by Littlewood et al. [12], and the 24.8 points after one year reported by Engebretsen et al. [13], all in comparable populations. It is, however, smaller than the 32.7 and 37.2 points (control and intervention group, respectively) after 12 weeks 
343 reported by Bal et al. [15]. This dissimilarity to the current and previous studies $[12,13,18]$ could

344 be explained by a seemingly shorter symptom duration in the population studied by Bal et al.

345 [15], as compared to our study and previous studies [12,13,18]. In summary, the results of

346 conservative treatment in this study are fairly encouraging, with mean improvements in SPADI

347 scores that are comparable to those found in previous trials $[12,13,18]$. This is, however, in slight

348 contrast to the fact that only $25 \%$ of the patients considered themselves fully recovered or much

349 improved at follow-up and median follow-up SPADI scores of 25 points [IQR: 8; 48], revealing

350 room for improvement in current care.

\section{Objective versus subjective outcomes}

353

354

355

356

357

358

359

360

361

362

363

364
In this study of patients with SIS who underwent conservative treatment in secondary care, patient-reported outcomes improved significantly while objective outcomes did not. Considering the lack of relationship between objective and subjective outcomes in this population [3], this inconsistency might not be surprising. However, these findings further suggests that the focus of treatment has, rightfully, been on the subjective symptoms, which are considered cardinal in SIS [2], while strength improvements have not necessarily been the focus of rehabilitation. In addition, the observed inconsistency is also likely affected by the larger effect of the non-specific parts of treatment (i.e. placebo) on subjective outcomes compared to objective outcomes, as has previously been described by Hróbjartsson \& Gøtzsche [29]. This, combined with the knowledge that objective impairment measures and subjective outcomes seems to measure different constructs [3], underlines the importance of including both when evaluating treatment effect. 


\section{Rehabilitation Parameters and relation to outcomes}

366 The relationship between specific rehabilitation parameters and the clinical outcomes of

367 treatment was limited in the current study, indicating that core clinical outcomes are not

368 sufficiently addressed in current care. Accordingly, only two statistically significant associations

369 were identified, with the effects $+0.7 \mathrm{~N}$ increase in external rotation strength per physiotherapy

370 session ( $95 \% \mathrm{CI} 0.0$ to $1.3, \mathrm{p}=0.046)$ and -0.2 points decrease on $0-10$ NRS per 1,000 minutes of

371 patient-reported exercise time $(-0.4$ to $0.0, \mathrm{p}=0.048)$. In this context, it should be noted that the

372 large number of regression analyses performed increases the risk of a type 1 error. In fact,

373 adjusting for this using a Bonferroni correction would mean that no relationships would come

374 out as significant. Therefore, considering the limited gains per session or 1,000 minutes, the

375 borderline significance of these associations and the risk of type 1 errors, the relationship

376 between the specific rehabilitation parameters investigated in the current study and the clinical

377 outcomes cannot be considered clinically relevant.

\section{Strengths and limitations}

380

381

382

383

384

385

386

An important strength to the current study is the application of a consecutive sampling strategy, which increases the generalizability of the results. It should be noted, however, that one third of the conservatively-treated SIS-patients did not participate in follow-up assessment of strength and ROM. This might reduce the internal validity of the results due to selection bias in case of systematic differences between patients who did and did not participate in follow-up assessment. However, when comparing baseline and rehabilitation parameters, we found that only average pain at baseline $(p=0.02$, see Table 2$)$ and the total time spent on exercises $(p=0.045$, see Table 
387 4) differed significantly between groups, indicating that the risk of selection bias is minor,

388 though it should still be considered when interpreting the findings of the current study.

In the regression analyses, we only adjusted for baseline scores of the relevant variable, and not for other covariates, such as duration of symptoms. While this leaves a risk of residual confounding, post hoc analyses including duration of symptoms as a covariate in all regression analyses did not have a relevant impact on the results, clearly indicating that symptom duration did not confound the relationship between rehabilitation parameters and outcomes in this study.

For the variables time spent on exercises and number of physiotherapy sessions, only exercise time and number of sessions were recorded. Therefore, one could argue that important differences in the content of exercises and physiotherapy sessions have been ignored, as such information would provide additional insight into the relationships between treatment and outcome. There is also a risk of recall bias, especially related to the variable time spent on exercises. As studies have shown that, even in exercise diaries, patients vastly overestimate the amount of exercise they perform [30], this will in turn decrease the improvement in outcomes per reported exercise time. However, looking from a patient perspective, it seems relevant that the more time you put into performing exercises or the more physiotherapy sessions you attend, the better the outcome, at least when focusing on main outcomes such as SPADI and pain. Nevertheless, based on the inherent limitations to the applied method, it should be stressed that the exploratory finding of no relevant relationship between patient-reported specific rehabilitation parameters and treatment outcomes should be interpreted with caution. Rather, the results presented here serve as a clear indication that more research is needed to investigate how to address specific clinical outcomes, and to what extent these outcomes are addressed in current 
410 care, using prospectively recorded and valid measures of adherence to specific exercises and the

411 content of physiotherapy.

\section{Conclusion}

414 In conclusion, conservatively-treated patients with SIS did not improve in objective clinical

415 outcomes, but significant improvements were seen in patient-reported outcomes. Furthermore,

416 no relevant relationships between specific rehabilitation parameters and improvements in

417 outcomes were identified, but these findings should be interpreted with caution. Collectively,

418 however, the findings of the current study indicate room for improvement of the current

419 rehabilitation of SIS, especially with regard to core clinical outcomes, such as strength and range

420 of motion.

421

422

\section{Acknowledgements}

424 The authors would like to thank the patients who participated in this study, and all the personnel 425 at the Sports Orthopedic Research Center - Copenhagen (SORC-C) for their assistance.

\section{$\underline{\text { References }}$}

428

1. van der Windt DA., Koes BW., de Jong BA., Bouter LM. 1995. Shoulder disorders in general practice: incidence, patient characteristics, and management. Annals of the rheumatic diseases 54:959964. 
432 2. Roach KE., Budiman-Mak E., Songsiridej N., Lertratanakul Y. 1991. Development of a shoulder pain

433

434

436

437

438

439

440

441

442

443

444

445

446

447

448

449

450

451

452

453

454 and disability index. Arthritis Care and Research: The Official Journal of the Arthritis Health Professions Association 4:143-149.

3. Clausen MB., Witten A., Holm K., Christensen KB., Attrup ML., Hölmich P., Thorborg K. 2017. Glenohumeral and scapulothoracic strength impairments exists in patients with subacromial impingement, but these are not reflected in the shoulder pain and disability index. $B M C$ Musculoskeletal Disorders 18. DOI: 10.1186/s12891-017-1667-1.

4. MacDermid JC., Ramos J., Drosdowech D., Faber K., Patterson S. 2004. The impact of rotator cuff pathology on isometric and isokinetic strength, function, and quality of life. Journal of shoulder and elbow surgery / American Shoulder and Elbow Surgeons ... [et al.] 13:593-598. DOI: $10.1016 / \mathrm{S} 1058274604001247$.

5. Diercks R., Bron C., Dorrestijn O., Meskers C., Naber R., de Ruiter T., Willems J., Winters J., van der Woude HJ. 2014. Guideline for diagnosis and treatment of subacromial pain syndrome: A multidisciplinary review by the Dutch Orthopaedic Association. Acta Orthopaedica 85:314-322. DOI: $10.3109 / 17453674.2014 .920991$.

6. Sundhedsstyrelsen. 2013. National klinisk retningslinje for diagnostik og behandling af patienter med udvalgte skulderlidelser (Høringsversion). København.

7. Akyol Y., Ulus Y., Durmus D., Canturk F., Bilgici A., Kuru O., Bek Y. 2012. Effectiveness of microwave diathermy on pain, functional capacity, muscle strength, quality of life, and depression in patients with subacromial impingement syndrome: a randomized placebo-controlled clinical study. Rheumatology International 32:3007-3016. DOI: 10.1007/s00296-011-2097-2.

8. Dilek B., Gulbahar S., Gundogdu M., Ergin B., Manisali M., Ozkan M., Akalin E. 2016. Efficacy of Proprioceptive Exercises in Patients with Subacromial Impingement Syndrome: A Single-Blinded 
455

456

457

458

459

460

461

462

463

464

465

466

467

468

469

470

471

472

473

474

475

476

477

478

Randomized Controlled Study. American Journal of Physical Medicine \& Rehabilitation 95:169182. DOI: 10.1097/PHM.0000000000000327.

9. Lombardi I., Magri AG., Fleury AM., Da Silva AC., Natour J. 2008. Progressive resistance training in patients with shoulder impingement syndrome: a randomized controlled trial. Arthritis and Rheumatism 59:615-622. DOI: 10.1002/art.23576.

10. Başkurt Z., Başkurt F., Gelecek N., Özkan MH. 2011. The effectiveness of scapular stabilization exercise in the patients with subacromial impingement syndrome. Journal of Back and Musculoskeletal Rehabilitation 24:173-179. DOI: 10.3233/BMR-2011-0291.

11. Moezy A., Sepehrifar S., Solaymani Dodaran M. 2014. The effects of scapular stabilization based exercise therapy on pain, posture, flexibility and shoulder mobility in patients with shoulder impingement syndrome: a controlled randomized clinical trial. Medical Journal of the Islamic Republic of Iran 28:87.

12. Littlewood C., Bateman M., Brown K., Bury J., Mawson S., May S., Walters SJ. 2015. A self-managed single exercise programme versus usual physiotherapy treatment for rotator cuff tendinopathy: A randomised controlled trial (the SELF study). Clinical Rehabilitation. DOI: $10.1177 / 0269215515593784$.

13. Engebretsen K., Grotle M., Bautz-Holter E., Ekeberg OM., Juel NG., Brox JI. 2011. Supervised exercises compared with radial extracorporeal shock-wave therapy for subacromial shoulder pain: 1-year results of a single-blind randomized controlled trial. Physical therapy 91:37-47. DOI: 10.2522/ptj.20090338.

14. Yiasemides R., Halaki M., Cathers I., Ginn KA. 2011. Does passive mobilization of shoulder region joints provide additional benefit over advice and exercise alone for people who have shoulder pain and minimal movement restriction? A randomized controlled trial. Physical Therapy 91:178-189. DOI: 10.2522/ptj.20100111. 
479 15. Bal A., Eksioglu E., Gurcay E., Gulec B., Karaahmet O., Cakci A. 2009. Low-level laser therapy in 480 subacromial impingement syndrome. Photomedicine and Laser Surgery 27:31-36. DOI: 10.1089/pho.2007.2222.

16. McClure PW., Bialker J., Neff N., Williams G., Karduna A. 2004. Shoulder function and 3-dimensional kinematics in people with shoulder impingement syndrome before and after a 6-week exercise program. Physical therapy 84:832-848.

17. Holmgren T., Björnsson Hallgren H., Öberg B., Adolfsson L., Johansson K. 2012. Effect of specific exercise strategy on need for surgery in patients with subacromial impingement syndrome: randomised controlled study. BMJ (Clinical research ed.) 344:e787.

18. Bennell K., Wee E., Coburn S., Green S., Harris A., Staples M., Forbes A., Buchbinder R. 2010. Efficacy of standardised manual therapy and home exercise programme for chronic rotator cuff disease: randomised placebo controlled trial. BMJ (Clinical research ed.) 340:c2756.

19. Maenhout AG., Mahieu NN., De Muynck M., De Wilde LF., Cools AM. 2013. Does adding heavy load eccentric training to rehabilitation of patients with unilateral subacromial impingement result in better outcome? A randomized, clinical trial. Knee surgery, sports traumatology, arthroscopy: official journal of the ESSKA 21:1158-1167. DOI: 10.1007/s00167-012-2012-8.

20. Galace de Freitas D., Marcondes FB., Monteiro RL., Rosa SG., Maria de Moraes Barros Fucs P., Fukuda TY. 2014. Pulsed electromagnetic field and exercises in patients with shoulder impingement syndrome: a randomized, double-blind, placebo-controlled clinical trial. Archives of Physical Medicine and Rehabilitation 95:345-352. DOI: 10.1016/j.apmr.2013.09.022.

21. Ingwersen KG., Jensen SL., Sørensen L., Jørgensen HR., Christensen R., Søgaard K., Juul-Kristensen B. 2017. Three Months of Progressive High-Load Versus Traditional Low-Load Strength Training Among Patients With Rotator Cuff Tendinopathy: Primary Results From the Double-Blind 
502

503

504

505

506

507

508

509

510

511

512

513

514

515

516

517

518

519

520

521

522

523

Randomized Controlled RoCTEx Trial. Orthopaedic Journal of Sports Medicine

5:2325967117723292. DOI: 10.1177/2325967117723292.

22. Struyf F., Nijs J., Mollekens S., Jeurissen I., Truijen S., Mottram S., Meeusen R. 2013. Scapular-focused treatment in patients with shoulder impingement syndrome: a randomized clinical trial. Clinical Rheumatology 32:73-85. DOI: 10.1007/s10067-012-2093-2.

23. Braunholtz DA., Edwards SJ., Lilford RJ. 2001. Are randomized clinical trials good for us (in the short term)? Evidence for a "trial effect." Journal of Clinical Epidemiology 54:217-224.

24. Christiansen DH., Frost P., Frich LH., Falla D., Svendsen SW. 2016. The Use of Physiotherapy among Patients with Subacromial Impingement Syndrome: Impact of Sex, Socio-Demographic and Clinical Factors. PloS One 11:e0151077. DOI: 10.1371/journal.pone.0151077.

25. Hopman K., Lukersmith S., McColl A., Vine K. 2013. Clinical Practice Guidelines for the Management of Rotator Cuff Syndrome in the Workplace. Port Macquarie, Australia: University of South Wales.

26. Michener LA., Walsworth MK., Doukas WC., Murphy KP. 2009. Reliability and diagnostic accuracy of 5 physical examination tests and combination of tests for subacromial impingement. Archives of physical medicine and rehabilitation 90:1898-1903. DOI: 10.1016/j.apmr.2009.05.015.

27. Christiansen DH., Andersen JH., Haahr JP. 2013. Cross-cultural adaption and measurement properties of the Danish version of the Shoulder Pain and Disability Index. Clinical rehabilitation 27:355-360. DOI: 10.1177/0269215512456220.

28. Kolber MJ., Vega F., Widmayer K., Cheng M-SS. 2011. The reliability and minimal detectable change of shoulder mobility measurements using a digital inclinometer. Physiotherapy theory and practice 27:176-184. DOI: 10.3109/09593985.2010.481011. 
524 29. Hróbjartsson A., Gøtzsche PC. 2001. Is the placebo powerless? An analysis of clinical trials comparing 525 placebo with no treatment. The New England Journal of Medicine 344:1594-1602. DOI: 10.1056/NEJM200105243442106.

527 30. Rathleff MS., Bandholm T., McGirr KA., Harring SI., Sørensen AS., Thorborg K. 2016. New exercise528 integrated technology can monitor the dosage and quality of exercise performed against an elastic resistance band by adolescents with patellofemoral pain: an observational study. Journal of Physiotherapy 62:159-163. DOI: 10.1016/j.jphys.2016.05.016. 


\section{Table 1 (on next page)}

Interview regarding treatment and rehabilitation since baseline 
Table 1 Interview regarding treatment and rehabilitation since baseline

\begin{tabular}{lll}
\hline & Question \\
\hline Shoulder surgery & Q1 $\begin{array}{l}\text { "Have you had shoulder surgery since your baseline assessment } \\
\text { six months ago?" }\end{array}$ \\
\hline Corticosteroid injection & Q2 & $\begin{array}{l}\text { "Have you had a corticosteroid injection for your shoulder } \\
\text { disorder since baseline, including the day of the baseline } \\
\text { examination? If yes, how many times?" }\end{array}$ \\
\hline Physiotherapy & Q3 $\begin{array}{l}\text { "Have you, since the baseline examination, seen a } \\
\text { physiotherapist for your shoulder disorder? If yes, how many } \\
\text { times?" }\end{array}$ \\
\hline Exercises & Q4 $\begin{array}{l}\text { "Have you been doing home-based (or non-supervised) exercises } \\
\text { for your shoulder disorder since baseline?" } \\
\text { "For how many weeks in total since baseline have you actively } \\
\text { been doing these exercises" }\end{array}$ \\
Q6 & $\begin{array}{l}\text { "During these weeks, how much time (in minutes) did you in } \\
\text { average use per week on the exercises?" }\end{array}$
\end{tabular}




\section{Table 2 (on next page)}

Baseline characteristics for conservatively treated patients, separately for those who participated in follow-up assessment and those who did not. 
Table 2 Baseline characteristics for conservatively treated patients, separately for those who participated in follow-up assessment and those who did not.

\begin{tabular}{|c|c|c|c|}
\hline & $\begin{array}{c}\text { Conservatively } \\
\text { treated } \\
(\mathrm{n}=35)\end{array}$ & $\begin{array}{c}\text { Participated in } \\
\text { follow-up assessment } \\
(\mathrm{n}=63) \\
\end{array}$ & $P=$ \\
\hline Age in years, mean $\pm S D$ & $51 \pm 15$ years & $56 \pm 13$ years & 0.14 \\
\hline Gender, \% females & $51 \% \quad(18$ of 35$)$ & $54 \% \quad(34$ of 63$)$ & 0.84 \\
\hline Affected side, $\%$ dominant side & $53 \% \quad(17$ of 32$)$ & $59 \% \quad(37$ of 63$)$ & 0.66 \\
\hline Sick Leave, $\%$ on sick leave at baseline & $12 \% \quad(4$ of 33$)$ & $8 \% \quad(5$ of 62$)$ & 0.72 \\
\hline Duration of symptoms at baseline in $\%$ & & & 0.54 \\
\hline 0-1 month & $(0$ of 34$)$ & $3 \% \quad(2$ of 62$)$ & \\
\hline 1-3 months & $21 \% \quad(7$ of 34$)$ & $16 \% \quad(10$ of 62$)$ & \\
\hline 3-6 months & $18 \% \quad(6$ of 34$)$ & $26 \% \quad(16$ of 62$)$ & \\
\hline$>6$ months & $62 \% \quad(21$ of 34$)$ & $55 \% \quad(34$ of 62$)$ & \\
\hline \multicolumn{4}{|l|}{ Baseline values } \\
\hline SPADI Score, median [IQR] & $\mathrm{n}=34 \quad 61[43 ; 73]$ & $\mathrm{n}=63 \quad 54[38 ; 70]$ & 0.17 \\
\hline External rot. strength, Newton, median [IQR] & $\mathrm{n}=24 \quad 59[36 ; 72]$ & $\mathrm{n}=56 \quad 52[34 ; 86]$ & 0.77 \\
\hline Abduction strength, Newton, median [IQR] & $\mathrm{n}=25 \quad 55[37 ; 92]$ & $\mathrm{n}=56 \quad 54[36 ; 90]$ & 0.96 \\
\hline Abduction ROM, degrees, median [IQR] & $\mathrm{n}=27 \quad 101[90 ; 140]$ & $\mathrm{n}=58 \quad 120[87 ; 154]$ & 0.30 \\
\hline Average pain, median [IQR] & $\mathrm{n}=31 \quad 4[2.5 ; 5]$ & $\mathrm{n}=60 \quad 3[1.6 ; 4]$ & 0.02 \\
\hline Pain during external rot. strength, median [IQR] & $\mathrm{n}=24 \quad 4[1 ; 6]$ & $\mathrm{n}=56 \quad 4[2 ; 6]$ & 0.63 \\
\hline Pain during abduction strength, median [IQR] & $\mathrm{n}=24 \quad 4[0.3 ; 7.8]$ & $\mathrm{n}=56 \quad 3[1 ; 7]$ & 0.95 \\
\hline Pain during abduction ROM, median [IQR] & $\mathrm{n}=27 \quad 5[5 ; 8]$ & $\mathrm{n}=58 \quad 5.5[3 ; 8]$ & 0.06 \\
\hline
\end{tabular}




\section{Table 3(on next page)}

Baseline, follow-up and change score for SPADI, strength, ROM and pain variables in conservatively treated patients who participated in follow-up assessment. 
Table 3 Baseline, follow-up and change score for SPADI, strength, ROM and pain variables in conservatively treated patients who participated in follow-up assessment.

\section{Outcomes}

Normality test p-value change Effect Size

Mean (SD) Median [IQR]

SPADI score,

0 -100 points $(n=63)$

$\begin{array}{lrcccc}\text { Baseline } & 54 \pm 20 & 54[38 ; 70] & \text { Normal } & & \\ \text { Follow-up } & 31 \pm 26 & 25[8 ; 48] & \text { Non-norm. } & & \\ \text { Change } & -23 \pm 24 & -19[-39 ;-3] & \text { Normal } & <0.001 & -0.53\end{array}$

\section{External rot. strength,}

Newton $(n=56)$

Baseline

Follow-up

Change

\section{Abduction strength,}

\section{Newton $(n=56)$}

Baseline

Follow-up

Change

\section{Abduction ROM degrees $(n=57)$

Baseline
Follow-up
Change

\section{Average pain,}

0 -10 points $(n=60)$

Baseline

Follow-up

Change

\section{Pain during}

external rot. strength,

$0-10$ points $(n=56)$

Baseline

Change

\section{Pain during}

abduction strength,

0 -10 points $(n=56)$

Baseline

Follow-up

Change

$\begin{array}{rrl}62 \mathrm{~N} \pm 37 & 52 \mathrm{~N}[34 ; 84] & \text { Non-norm. } \\ 65 \mathrm{~N} \pm 35 & 56 \mathrm{~N}[44 ; 79] & \text { Non-norm. } \\ 3 \mathrm{~N} \pm 25 & 2 \mathrm{~N}[-9 ; 17] & \text { Non-norm. }\end{array}$

0.337

0.09

$\begin{array}{rcc}120^{\circ} \pm 40 & 121^{\circ}[93 ; 154] & \text { Normal } \\ 128^{\circ} \pm 35 & 137^{\circ}[107 ; 153] & \text { Non-norm. } \\ 8^{\circ} \pm 41 & -1^{\circ}[-12 ; 30] & \text { Non-norm. }\end{array}$

.324

0.09

$\begin{array}{rcc}3,0 \pm 1,5 & 3,0[1,8 ; 4] & \text { Normal } \\ 1,8 \pm 2,1 & 1,0[0 ; 3] & \text { Non-norm. } \\ -1,2 \pm 1,9 & -1,5[-2,5 ; 0] & \text { Normal }\end{array}$

$<0.001$

$-0.39$ 
Pain during

abduction ROM,

0 -10 points $(n=57)$

\begin{tabular}{lccccc} 
Baseline & $5,5 \pm 2,5$ & $6,0[3 ; 8]$ & Non-norm. & & \\
Follow-up & $3,0 \pm 3$ & $2,0[1 ; 5]$ & Non-norm. & & $<0.001$ \\
Change & $-2,3 \pm 3,5$ & $-1,0[-5 ; 0]$ & Normal & & -0.38 \\
& & & & & \\
\hline
\end{tabular}




\section{Table 4 (on next page)}

Specific rehabilitation parameters in the conservatively treated patients, separately for those who participated in follow-up assessment and those who did not.

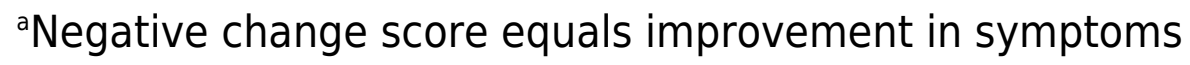


Table 4 Specific rehabilitation parameters in the conservatively treated patients, separately for those who participated in follow-up assessment and those who did not.

\begin{tabular}{|c|c|c|c|c|}
\hline \multirow{2}{*}{ Number of corticosteroid, by group } & \multicolumn{2}{|c|}{$\begin{array}{c}\begin{array}{c}\text { Not participated in } \\
\text { follow-up assessment } \\
(\mathrm{n}=35)\end{array} \\
\end{array}$} & $\begin{array}{c}\text { Participated in } \\
\text { follow-up assessment } \\
(\mathrm{n}=63)\end{array}$ & \multirow[t]{2}{*}{$P=$} \\
\hline & & & & \\
\hline 0 injection & $46 \%$ & $(16$ of 35$)$ & $38 \% \quad(24$ of 63$)$ & 0.16 \\
\hline 1 injection & $31 \%$ & (11 of 35) & $41 \% \quad(26$ of 63$)$ & \\
\hline 2 injections & $9 \%$ & (3 of 35) & $18 \% \quad(11$ of 63$)$ & \\
\hline 3 injections & $6 \%$ & (2 of 35) & $3 \% \quad(2$ of 63$)$ & \\
\hline 4 injections & $6 \%$ & (2 of 35) & & \\
\hline 5 injections & $3 \%$ & (1 of 35) & & \\
\hline Physiotherapy, \%yes & $57 \%$ & $(20$ of 35$)$ & $75 \% \quad(47$ of 63$)$ & 0.11 \\
\hline Number of Physio-Sessions, median [IQR] & 2 & {$[0 ; 12]$} & $5[0 ; 11]$ & 0.26 \\
\hline \multicolumn{5}{|l|}{ Grouped by number of Physio-Sessions } \\
\hline 0 sessions & $32 \%$ & $(15$ of 35$)$ & $26 \% \quad(16$ of 62$)$ & 0.37 \\
\hline 1 to 5 sessions & $24 \%$ & (7 of 35) & $26 \% \quad(16$ of 62$)$ & \\
\hline 6 to 10 sessions & $18 \%$ & (4 of 35) & $21 \% \quad(13$ of 62$)$ & \\
\hline$>10$ sessions & $27 \%$ & (9 of 35) & $27 \% \quad(17$ of 62$)$ & \\
\hline Home Exercises, yes n(\%) & $76 \%$ & $(26$ of 34$)$ & $87 \% \quad(55$ of 63$)$ & 0.25 \\
\hline Total minutes of home exercises, median [IQR] & 600 & {$[0 ; 1560]$} & $1040[220 ; 2700]$ & 0.045 \\
\hline
\end{tabular}

1 


\section{Table 5(on next page)}

Regression analyses. The influence of specific rehabilitation parameters on the change in core clinical outcomes. Adjusted for baseline value of the relevant outcome. 
Table 5 Regression analyses. The influence of specific rehabilitation parameters on the change in core clinical outcomes. Adjusted for baseline value of the relevant outcome.

\begin{tabular}{|c|c|c|c|c|}
\hline te & $\begin{array}{c}\text { SPADI }^{\mathrm{a}} \\
\text { (Points: } 0-100)\end{array}$ & $\begin{array}{c}\text { External rot. } \\
\text { Strength } \\
\text { (Newton) } \\
\end{array}$ & $\begin{array}{l}\text { Abduction } \\
\text { strength } \\
\text { (Newton) } \\
\end{array}$ & $\begin{array}{c}\text { Abduction } \\
\text { ROM } \\
\text { (Degrees) } \\
\end{array}$ \\
\hline \multicolumn{5}{|l|}{ Time spent on home exercises } \\
\hline $\mathrm{B}(\Delta$ outcome per $1.000 \mathrm{~min})$ & -2.3 & 0.3 Newton & -0.6 Newton & $2^{\circ}$ \\
\hline$(95 \% \mathrm{CI})$ & $(-4.8$ to 0.1$)$ & $(-2.1$ to 2.8$)$ & $(-3.4$ to 2.2$)$ & $(-2$ to 5$)$ \\
\hline$p$-value & 0.058 & 0.779 & 0.654 & 0.370 \\
\hline \multicolumn{5}{|l|}{ Number of Physio-Sessions } \\
\hline B ( $\Delta$ outcome per session $)$ & 0.3 & 0.7 & 0.0 & $0^{\circ}$ \\
\hline$(95 \% \mathrm{CI})$ & $(-0.3$ to 0.8$)$ & (0.0 to 1.3$)$ & $(-0.8$ to 0.8$)$ & $(-1$ to 1$)$ \\
\hline$p$-value & 0.385 & 0.046 & 0.984 & 0.962 \\
\hline \multicolumn{5}{|l|}{ Number of Steroid injections } \\
\hline $\mathrm{B}(\Delta$ outcome per injection $)$ & 4.0 & 3.8 & 3.4 & $0^{\circ}$ \\
\hline$(95 \% \mathrm{CI})$ & $(-3.0$ to 11.1$)$ & $(-4.1$ to 11.6$)$ & $(-5.6$ to 12.4$)$ & $(-11$ to 12$)$ \\
\hline$p$-value & 0.256 & 0.338 & 0.453 & 0.944 \\
\hline
\end{tabular}

${ }^{\mathrm{a}} 0$ is best, 100 is worst. Negative change score equals improvement in symptoms

1 


\section{Table 6(on next page)}

Regression analyses. Influence of specific rehabilitation parameters on the change in pain outcomes in patients participating in follow-up assessment. Adjusted for baseline value of relevant outcome. 
Table 6 Regression analyses. Influence of specific rehabilitation parameters on the change in pain outcomes in patients participating in follow-up assessment. Adjusted for baseline value of relevant outcome.

\section{Average Pain during last week $^{\mathbf{a}}$ (0-10 points)}

Pain during tests (NPRS, 0-10 points)

External rot. Abduction Abduction Strength test $^{\mathrm{a}} \quad$ strength test $^{\mathrm{a}} \quad$ ROM test ${ }^{\mathrm{a}}$

\section{Time spent on home exercises}

$$
-0.2
$$

$(-0.4$ to 0.1$)$

0.232
$(-0.4$ to 0.1$)$
0.236

$-0.2$

(-0.6 to 0.1$)$

0.138
B ( $\triangle$ outcome per $1.000 \mathrm{~min})$

$(95 \% \mathrm{CI})$

p-value

$$
-0.2
$$

$(-0.4$ to 0.0$)$

$$
0.048
$$

0.0

$(-0.0$ to 0.1$)$

0.726

p-value
B ( $\Delta$ outcome per session)

$(95 \% \mathrm{CI})$

\section{Number of Steroid injections}

B ( $\Delta$ outcome per injection)

$(95 \% \mathrm{CI})$

p-value

${ }^{\mathrm{a}} 0$ is no pain, 10 is worst pain. Negative change score equals improvement in symptoms 


\title{
Figure 1 (on next page)
}

\author{
Flow-chart
}




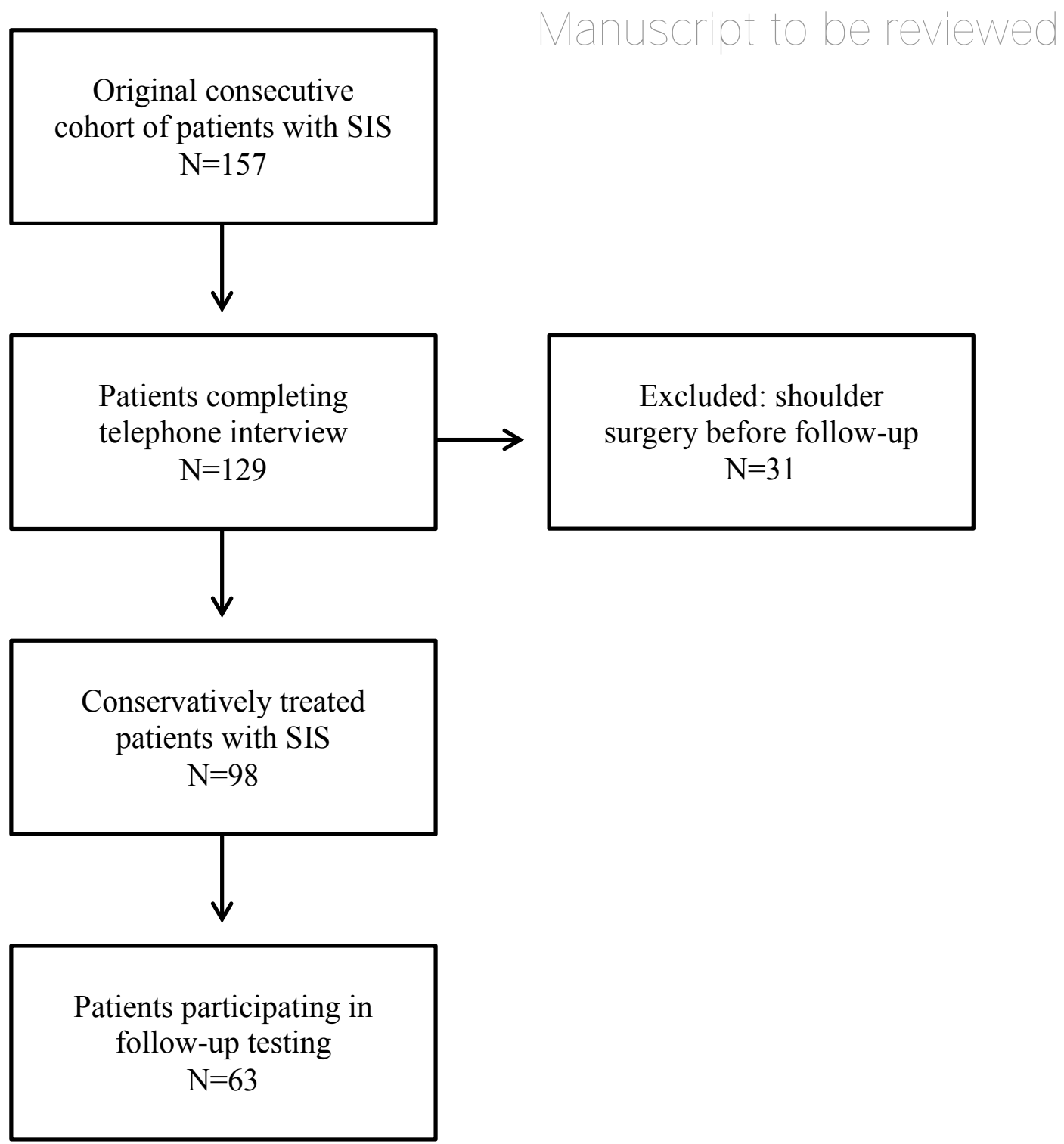




\section{Figure 2 (on next page)}

\section{Global impression of change since baseline}

Proportion of patients who participated in the follow-up assessment who reported being 1 ) fully cured or much improved, 2) improved, 3) small improvement, 4) no change, 5) little worse, 6) worse or 7) much worse at follow-up $(n=63)$. 


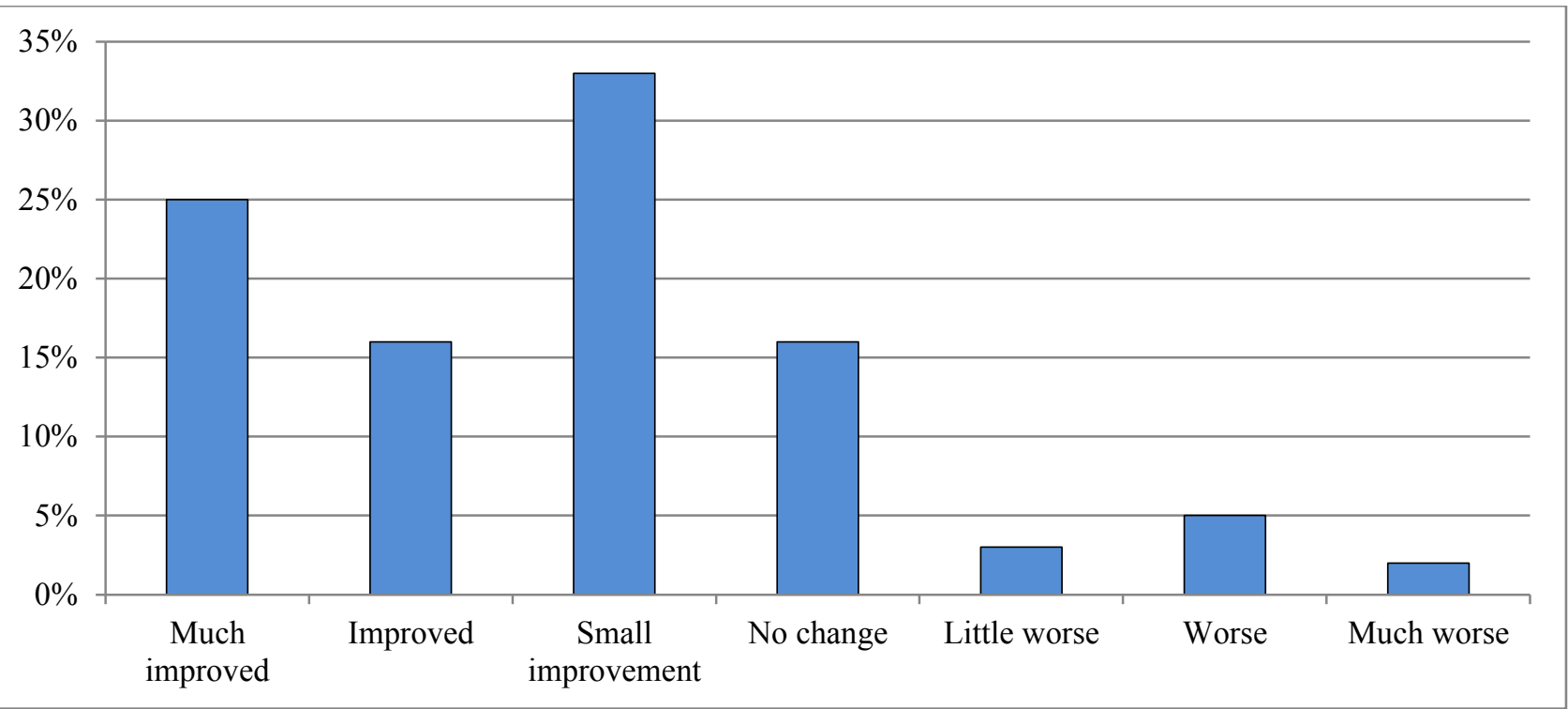




\section{Figure 3 (on next page)}

Severity at follow-up

Distribution of patient-reported severity of the shoulder disorder at follow-up, in patients who participated in the follow-up assessment $(n=63)$. Severity was scored on a 1 to 5 numeric rating scale ( $1=$ very mild and $5=$ very severe). 


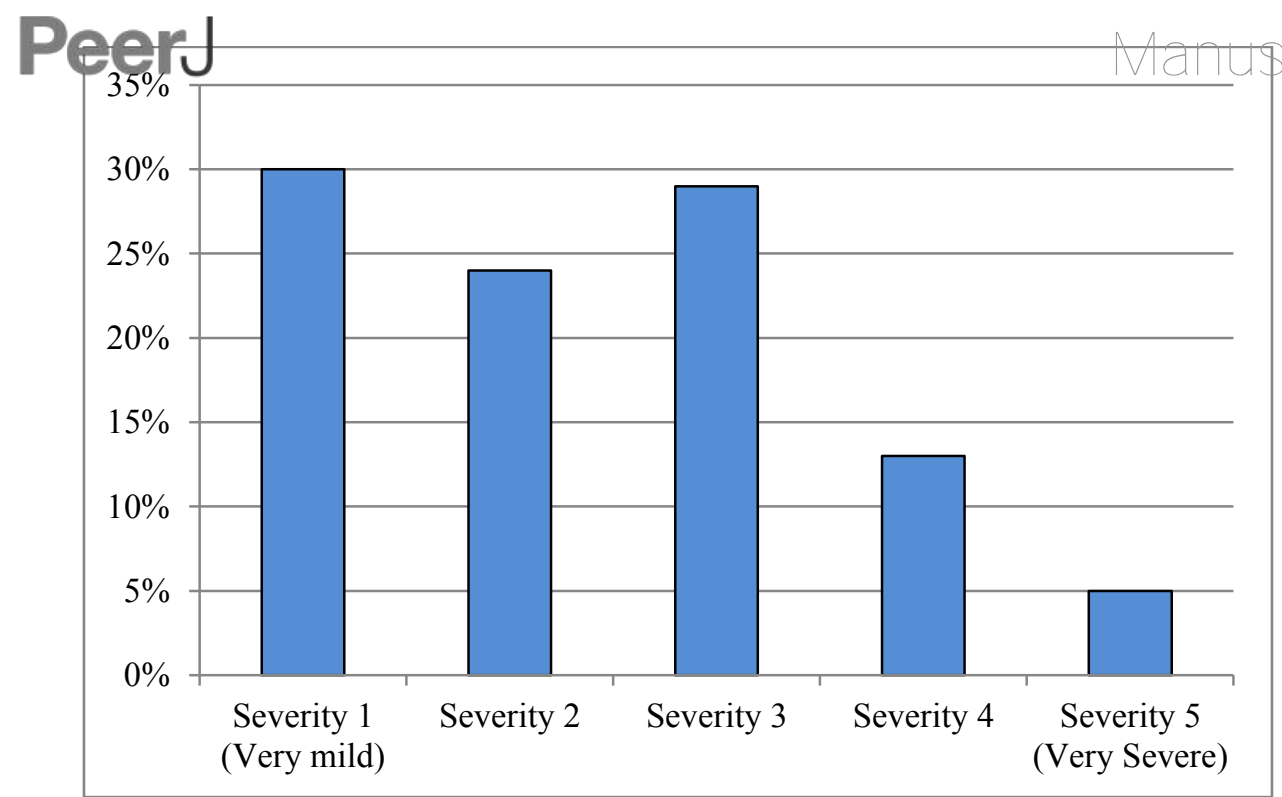

